\title{
EL DESARROLLO TECNOLÓGICO DE LA SOCIEDAD Y SUS INCIDENCIAS EN EL PENSAMIENTO LÓGICO MATEMÁTICO TECHNOLOGICAL DEVELOPMENT IN THE SOCIETY AND ITS EFFECTS ON THE MATHEMATICAL LOGICAL THINKING
}

\author{
Volumen 14, Número 2 \\ Mayo - Agosto \\ pp. 1-18
}

Este número se publicó el 30 de mayo de 2014

Rafael Bermúdez Tacunga

Revista indizada en REDALYC, $\underline{\text { SCIELO }}$

Revista distribuida en las bases de datos:

CATÁLOGO DE LATINDEX, IRESIE, CLASE, DIALNET, DOAJ, E-REVIST@S, SHERPA/ROMEO, QUALIS, MIAR

Revista registrada en los directorios:

ULRICH'S, REDIE, RINACE, OEI, MAESTROTECA, PREAL, CLASCO 


\title{
EL DESARROLLO TECNOLÓGICO DE LA SOCIEDAD Y SUS INCIDENCIAS EN EL PENSAMIENTO LÓGICO MATEMÁTICO TECHNOLOGICAL DEVELOPMENT IN THE SOCIETY AND ITS EFFECTS ON THE MATHEMATICAL LOGICAL THINKING
}

\begin{abstract}
Rafael Bermúdez Tacunga ${ }^{1}$
Resumen: Durante la última década, la sociedad del conocimiento ha evolucionado de manera acelerada motivando el surgimiento de herramientas tecnológicas que han incidido en diversos aspectos que integran la sociedad humana, entre los cuales se puede destacar el estándar de educación y formación que obtienen los estudiantes en las instituciones educativas de nivel superior, especialmente. Estos avances tecnológicos han provocado una suerte de adaptación en los estudiantes, quienes se ven obligados a depender de máquinas y equipos con tecnología actualizada para ser competitivos en las aulas de clases y, luego, en el exigente mercado laboral, dependencia que, indudablemente, es un factor que incide en la autonomía que poseemos las personas para formular, enfrentar y solucionar problemas relacionados con la ciencia matemática. En virtud de lo anterior, en el presente ensayo se efectúa un análisis del proceso del avance tecnológico que ha evidenciado la sociedad y sus implicaciones en el desarrollo del pensamiento lógico matemático de los estudiantes del nivel superior de estudios, para efectos de establecer la pertinencia del uso de equipos tecnológicos en la formulación y resolución de problemas matemáticos que impliquen deducciones lógicas para plantear los algoritmos alternativos de solución a los mismos.
\end{abstract}

Palabras clave: DESARROLLO TECNOLÓGICO, MATEMÁTICA, PENSAMIENTO LÓGICO, FORMULACIÓN DE PROBLEMAS, RESOLUCIÓN DE PROBLEMAS, EDUCACIÓN SUPERIOR

\begin{abstract}
During the last decade, the knowledge society has evolved rapidly encouraging the emergence of technological tools that have affected various aspects that make up human society, among which we can highlight the standard of education and training to get students in the higher educational institutions, especially. These technological advances have led to a kind of adaptation to the students, who are forced to rely on machines and equipment with updated technology to be competitive in the classroom and later in the demanding labor market, dependence that is undoubtedly a factor which affects people we have to make, confront and solve problems related to mathematical science. In this paper, there is an analysis of the process of technological advance that has shown society and its implications in the development of logical mathematical level students study, for purposes of establishing the relevance of the use of technological equipment in formulating and solving mathematical problems involving logical deductions to propose alternative algorithms for solving the same.
\end{abstract}

Key words: TECHNOLOGICAL DEVELOPMENT, MATHEMATICS, THOUGHT LOGICAL, FORMULATION OF PROBLEMS, TROUBLESHOOTING, HIGHER EDUCATION

\footnotetext{
1 Docente de la Universidad Laica Eloy Alfaro de Manabí,

Ecuador.
}

Dirección electrónica: rafabermudez12@yahoo.com

Ensayo recibido: 11 de octubre, 2013

Devuelto para corrección: 11 de noviembre, 2013

Aprobado: 15 de mayo, 2014 


\section{Introducción}

La sociedad humana se ha visto afectada por diversos descubrimientos tecnológicos entre los cuales se destacan los relacionados con la comunicación interpersonal, o los utilizados para efectuar cálculos matemáticos, que de manera evidente han llegado para permanecer entre nosotros con notables tendencias al perfeccionamiento constante, originando consecuencias positivas y negativas para el desarrollo integral del ser humano.

El sistema educativo, como parte importante de la sociedad humana, no puede mantenerse ajeno a tal situación, pues sería negarle a los estudiantes tanto la posibilidad como el derecho que tienen de poder desempeñarse eficientemente en el entorno que los rodea, contradiciendo, de esta manera, los reales y magnos postulados de la educación escolarizada y la misión de las instituciones educativas de todo nivel.

Es innegable que existe una relación entre la tecnología y el desarrollo del pensamiento lógico matemático, que se presenta con efectos en los estudiantes, los cuales deben ser investigados y analizados de una forma técnica para establecer estrategias y procedimientos pedagógicos que permitan armonizar el uso de herramientas tecnológicas como las calculadoras científicas y programas informáticos con el mejoramiento permanente de las habilidades y capacidades que poseen los estudiantes para solucionar situaciones problemáticas numéricas que indiscutiblemente serán parte de los eventos que afrontarán en sus respectivas vidas cotidianas o laborales.

La experiencia adquirida como docente de Matemáticas durante 11 años, en el nivel de estudios superiores dentro de instituciones privadas y públicas, es la base para la realización del presente ensayo con el tema propuesto, y que tiene como finalidad incrementar el análisis científico sobre la conveniencia del uso de equipos tecnológicos en la formación de profesionales mediante el establecimiento de niveles de acceso en la solución de problemas de razonamiento lógicos numéricos.

\section{Desarrollo del tema}

\section{Proposición}

Las herramientas tecnológicas que se usan como elementos complementarios del proceso de enseñanza aprendizaje de la Matemática no contribuyen con el desarrollo del pensamiento lógico de los estudiantes. 


\section{Argumentos para la discusión}

Para efectos de iniciar el análisis y discusión sobre la conveniencia de la utilización de equipos tecnológicos en el proceso formativo profesional de los estudiantes universitarios, es necesario señalar ciertos conceptos y definiciones sobre las diferentes categorías que se exponen en el ensayo, como un requisito ineludible para un mejor entendimiento del entorno en el cual se plantea el debate y para mantener un desarrollo coherente de los criterios que se exponen.

\section{Concepto y definición de tecnología}

Tecnología es el conjunto de conocimientos técnicos y ordenados científicamente, que permiten diseñar y crear bienes y servicios que facilitan la adaptación al medio ambiente, y satisfacer tanto las necesidades esenciales como los deseos de la humanidad. Es una

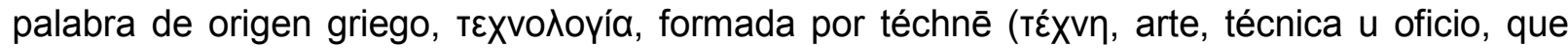

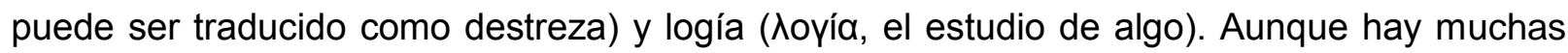
tecnologías muy diferentes entre sí, es frecuente usar el término en singular para referirse a una de ellas o al conjunto de todas.

Cuando se escribe con mayúscula, Tecnología, puede referirse tanto a la disciplina teórica que estudia los saberes comunes a todas las tecnologías como la educación tecnológica, la disciplina escolar abocada a la familiarización con las tecnologías más importantes; para los intereses del presente ensayo se señalará y entenderá como 'tecnología' a los equipos que se utilizan en los procesos de enseñanza aprendizaje de la comprensión y resolución de problemas matemáticos, para efectos de establecer la relación existente entre el uso de estos equipos con el pensamiento lógico matemático que deben desarrollar los estudiantes como base para la resolución de problemas numéricos relacionados con la carrera profesional que han escogido y posterior desempeño profesional laboral.

\section{Funciones de la tecnología}

Las funciones de la tecnología deben analizarse considerando dos perspectivas relacionadas con el tiempo; así se tiene que, en la prehistoria, las tecnologías fueron usadas para satisfacer necesidades esenciales (alimentación, vestimenta, vivienda, protección personal, relación social, comprensión del mundo natural y social), y en la historia también 
para obtener placeres corporales y estéticos (deportes, música, hedonismo en todas sus formas) y como medios para satisfacer deseos (simbolización de estatus, mejoramiento de procesos, etc.).

Caso especial resulta la función de la tecnología en la educación y formación de futuros profesionales, donde es estrictamente necesario que aprendan y dominen el manejo de equipos informáticos para un desempeño eficiente en el campo laboral, a tal punto, que en la mayoría de los casos se presenta una dependencia excesiva del ser humano hacia las herramientas tecnológicas de trabajo. Actualmente, no se puede concebir un profesional con un desempeño laboral eficiente sin conocimientos sobre el manejo y aplicación de programas y equipos tecnológicos que agilizan los procesos de ejecuciones de las tareas de tipo laboral.

Pese a lo expuesto, resulta importante plantearse como interrogante el nivel de tecnología que deben usar los estudiantes para que el desarrollo de su pensamiento lógico matemático no se exponga a una dependencia excesiva de estos, e influya en que, por motivos del acceso fácil a los equipos informáticos, los utilicen siempre, incluso para la realización de operaciones elementales de multiplicación y división de cantidades enteras, situación que se agrava por la extrema confianza que tienen los estudiantes en los resultados obtenidos mediante las máquinas. Generalmente, no comprueban mediante algún proceso adicional los resultados de las operaciones efectuadas, por considerar que mantienen un nivel incuestionable de acierto y, más aún, en ningún momento efectúan una validación lógica de los valores que se mantienen como solución del problema planteado.

Es importante recalcar que la tecnología mantiene ciertas funciones específicas, entre las cuales se destaca, a criterio del autor, la función simbólica de los objetos tecnológicos; esta situación se demuestra cuando la función principal de los objetos tecnológicos no es la de satisfacer las necesidades básicas de las personas y, más bien, se convierten en medios para establecer estatus social y relaciones de poder.

En los estudiantes universitarios este fenómeno se presenta de manera reiterada y se puede avizorar mediante las preferencias que tienen en la adquisición de equipos tecnológicos, como teléfonos celulares con alta tecnología y precios muy elevados, sobre la adquisición de equipos tecnológicos utilizados como complementos de los estudios superiores; la mayor cantidad de estudiantes poseen teléfonos móviles caros a pesar de no tener un computador o una calculadora científica para ser utilizada en sus estudios, lo cual 
sugiere que la función simbólica de la tecnología está superando a la función de aplicación técnica de la misma.

En estudios realizados se pudo observar que de cada 60 estudiantes pertenecientes a carreras administrativas, 52 poseen teléfonos celulares con alta tecnología, cuyos precios fluctúan entre 200 a 700 dólares norteamericanos, y no poseían un computador para sus estudios o una calculadora científica para efectuar cálculos numéricos.

El lector del presente ensayo se preguntará ¿cuál es el motivo de presentar esta información relacionada con la posesión de equipos tecnológicos? ¿Qué relación tiene lo expresado con el tema del ensayo que se expone?

El análisis efectuado sirve para comprender la lógica del pensamiento que poseen los estudiantes universitarios a la hora de adquirir herramientas tecnológicas para su uso particular, situación que, eventualmente y de la mano de factores como los medios de comunicación, la subcultura del consumo y la publicidad, entre otros posibles, se convierten en un problema para los estudios, y afectan de manera directa el desarrollo del proceso de enseñanza aprendizaje.

Observaciones realizadas en las aulas de clases, tomando como objeto de análisis a los 52 estudiantes identificados como tenedores de teléfonos con alta tecnología, permitieron corroborar en varias sesiones de trabajo que el $95 \%$ de los investigados no se concentraban en los temas tratados por el profesor y, precisamente, eran aquellos que mantenían prendidos sus equipos, lo cual les originaba evidentes desconcentraciones; el 5\% de estudiantes que poseen equipos celulares con alta tecnología y que están concentrados en clases, son aquellos que mantienen los equipos apagados durante el desarrollo de las clases. Esta situación se agudizó cuando los temas tratados implicaban necesariamente el uso del pensamiento lógico matemático.

Las valoraciones antes expuestas no tienen la intención de mostrar que las tecnologías son la causa de la desconcentración de los estudiantes durante el proceso de enseñanza aprendizaje, pero sí corroboran el supuesto de su incidencia.

\section{Relación de los estudios con los métodos de la tecnología}

Los métodos difieren según se trate de tecnologías de producción artesanal o industrial de artefactos, de prestación de servicios, de realización u organización de tareas de cualquier tipo, lo cual puede incidir en la clasificación de la tecnología aplicada con base en la 
complejidad que posee; esta afirmación es importante para la educación universitaria por cuanto es innegable que en muchas instituciones de estudios superiores no existe la suficiente tecnología que permita formar adecuadamente a los profesionales, lo cual evidentemente se constituye en una contradicción externa entre las exigencias del mercado laboral en relación con el manejo de equipos tecnológicos y la enseñanza que reciben los estudiantes respecto al uso de los equipos, lo que está en discusión en el presente ensayo es la utilización de equipos tecnológicos en situaciones educativas que impliquen uso del pensamiento lógico matemático.

La ineficiente capacitación en manejo de equipos tecnológicos que se utilizarán en futuras labores profesionales es una situación problema que se mantiene en los actuales momentos y que afecta, con mayor incidencia, a los estudiantes que por su condición socio económica no tienen en sus casas equipos de importante utilización como las computadoras.

Se pudo comprobar mediante visitas a las casas de los 60 estudiantes investigados, que 18 de ellos no tienen computadoras en sus casas $y$, precisamente, son ellos quienes mantienen mayores dificultades en el manejo de este tipo de equipos tecnológicos; sin embargo, mantienen niveles de razonamiento lógico superior a quienes poseen computadoras en sus casas, lo cual se evidencia mediante las puntuaciones más elevadas alcanzadas en los test de razonamiento numérico a los cuales fueron sometidos todos los estudiantes investigados.

Un método común a todas las tecnologías de fabricación es el uso de herramientas e instrumentos para la construcción de artefactos, en este uso definitivamente se plantean situaciones problemáticas que requieren una capacidad lógica numérica por parte de los profesionales formados en las universidades para solucionarlas. Las tecnologías de prestación de servicios, como el sistema de suministro eléctrico, hacen uso de instalaciones complejas a cargo de personal especializado. Las máquinas herramientas son combinaciones complejas de varias herramientas gobernadas (actualmente, muchas mediante computadoras) por información obtenida desde instrumentos, también incorporados en ellas.

Es notorio que existe una notable relación entre el pensamiento lógico, la solución de problemas y la invención de artefactos; el enlace armónico de estas categorías debe ser uno de los objetivos prioritarios en la formación técnica humanista de los estudiantes universitarios; la invención de equipos tecnológicos para mejorar el desarrollo socio económico del entorno debe convertirse en una meta a conseguir mediante el desarrollo del 
razonamiento lógico. Como se evidencia, el razonamiento lógico matemático mantiene una correlación positiva alta con la creatividad de las personas y, por ende, con su nivel de invención de nuevos artículos o bienes que posteriormente sirven para cubrir necesidades que se presentan en las tareas cotidianas.

Según el científico I. Asimov (1958), inventar exigía trabajar duro y pensar firme. Por su parte, J.P. Guilford (1958), destacado estudioso de la psicología de la inteligencia, identifica como las principales destrezas de un inventor las incluidas en lo que denomina aptitudes de producción divergente. Guilford propone un modelo de inteligencia que permite su análisis factorial, cuyas dimensiones son tres:

1 Proceso intelectual: actividad que realiza una persona para convertir la información en conocimiento.

2 Producto intelectual: conformación u organización de la información según un orden de complejidad.

3 Contenido de información.

Es un modelo íntegro que considera tanto a la inteligencia como a la información para poder definir más el concepto de habilidad mental, que es el resultado de la combinación de un proceso, un producto y un contenido de información. Gracias a este modelo, es posible lograr aplicaciones para medir y desarrollar las habilidades intelectuales como herramientas fundamentales del aprendizaje. Toma como punto de partida un análisis de cómo actúa el sistema cognitivo al resolver problemas. Para ello, establece tres categorías, cuyas intercepciones dan lugar a cada aptitud:

Operaciones: Tipo de proceso intelectual (valoración, producción convergente, producción divergente, retención de memoria, registro de memoria, cognición).

Contenidos: Tipo de información con el cual se trabaja (visual, auditivo, simbólico, semántico, comportamental).

Productos: Forma que adopta la información en el procesamiento que el organismo hace de ella (unidades, clases, relaciones, sistemas, transformaciones, implicaciones).

\section{Tipos de tecnología}

El pensamiento lógico matemático es una facultad que le permite a una sociedad incrementar el desarrollo tecnológico que posee, y que se puede considerar como tecnología 
dura o tecnología blanda, dependiendo del campo de aplicación del cual se derive; muchas veces, la palabra tecnología se aplica a la informática, la micro eléctrica, el láser o a las actividades especiales, tecnologías que son consideradas como duras por el entorno social y son reconocidas como tal en función de las ciencias que la estructuran; generalmente, la sociedad reconoce como tecnologías duras a aquellas que se derivan de ciencias como la física, química y la informática,

Sin embargo, la mayoría de las definiciones que se conocen también permiten e incluyen a otras, a las que se suele denominar como blandas. Las tecnologías blandas -en las que su producto no es un objeto tangible, como los servicios- pretenden mejorar el funcionamiento de las instituciones $u$ organizaciones para el cumplimiento de sus objetivos. Dichas organizaciones pueden ser empresas industriales, comerciales o de servicio, instituciones con o sin fines de lucro, instituciones privadas o públicas, etc.

Entre las ramas de la tecnología llamadas blandas se destaca de manera evidente la educación (en lo que respecta al proceso de enseñanza), puesto que mediante el proceso de enseñanza aprendizaje se capacita a las personas en conocimientos técnicos que les permitirá crear bienes o servicios para satisfacer necesidades del entorno social donde se desenvuelven; igualmente, dentro de este tipo de tecnología se contempla la organización, la Administración, la Contabilidad y las Operaciones, la Logística de producción, el Mercadeo, la Estadística, la Psicología de las relaciones humanas y del trabajo, y el Desarrollo de programas de cómputo que utilizan las organizaciones. En términos generales, las tecnologías blandas se fundamentan en ciencias blandas, como la Sociología, la Pedagogía, la Economía o la Administración.

Existen otras clasificaciones de la tecnología que son muy reconocidas por la sociedad, entre las cuales se cita a las llamadas tecnologías apropiadas (Tecnología adecuada). Se considera que una tecnología es apropiada cuando tiene efectos beneficiosos sobre las personas y el medio ambiente. Aunque el tema es hoy objeto de intenso debate (y probablemente seguirá siéndolo por mucho tiempo), hay acuerdos bastante amplios y definidos por los integrantes de la sociedad respecto a las principales características que una tecnología debe tener para ser social y ambientalmente apropiada:

- No causar daño previsible a las personas, ni daño innecesario a las restantes formas de vida (animales y plantas). 
- No comprometer de modo irrecuperable el patrimonio natural de las futuras generaciones.

- Mejorar las condiciones básicas de vida de todas las personas, independientemente de su poder adquisitivo.

- No ser coercitiva y respetar los derechos y posibilidades de elección de sus usuarios voluntarios y de sus sujetos involuntarios.

- No tener efectos generalizados irreversibles, aunque estos parezcan a primera vista ser beneficiosos o neutros.

Los conceptos tecnologías apropiadas y tecnologías de punta son completamente diferentes. Las tecnologías de punta, término publicitario que enfatiza la innovación, son usualmente tecnologías complejas que hacen uso de muchas otras tecnologías más simples, y se encuentran presentes en los bienes y servicios que aplican situaciones noveles y mejoradas respecto de las existentes en su respectivo mercado de consumo.

Para efectos de ampliar el tema de discusión propuesto en el presente ensayo, es necesario incrementar las argumentaciones sobre el pensamiento lógico y exponer aportaciones de teóricos en relación con el tema que, sin lugar a dudas, son referencias científicas importantes que hay que considerarlas para un mejor sustento de las exposiciones de criterios que se efectúan

\section{Pensamiento lógico}

Cuando se asume algún concepto sobre pensamiento lógico, es necesario considerar que el acto de pensar es aquel que pone en funcionamiento el cerebro humano para permitirle conocer, imaginar, abstraer, analizar o comparar el mundo que lo rodea o inventarse fantasías.

El pensamiento lógico puede y debe ser desarrollado desde el proceso de enseñanza aprendizaje, como un requisito importante para el estudiante una vez egresado se desempeñe con eficiencia dentro del campo laboral, puesto que, no se concibe un profesional eficiente sin un sentido creativo y desarrollo lógico que les permita enfrentar situaciones problemáticas que se presentan cotidianamente y sobre las cuales debe asumir decisiones que tendrán un efecto sobre la organización en la que labora.

El desarrollo del pensamiento lógico requiere un entrenamiento y constante capacitación, las personas no nacemos con un sentido lógico de las cosas; el entrenamiento 
del pensamiento lógico, sobre todo el matemático, requiere concentración y tiempo de dedicación, por lo que, durante esta etapa de "gimnasia mental" que deben realizar los estudiantes, no es recomendable que utilicen equipos tecnológicos que generalmente se consideran como herramientas pedagógicas complementarias; la ejercitación del pensamiento lógico matemático de los estudiantes universitarios se la puede realizar con base en un sistema de tareas profesionalizantes que mantengan un nivel de complejidad ascendente y que implique situaciones problemáticas que los estudiantes puedan resolver sin necesidad de la aplicación de operaciones aritméticas o algebraicas que requieran fundamentos matemáticos importantes para su solución. Es necesario destacar que una forma de manifestación del pensamiento lógico es la capacidad de abstracción del individuo.

El doctor Miguel Palacios Frugone considera que los niños solo poseen pensamientos concretos: entienden lo que ven, por lo cual para comprender, por ejemplo, que dos más dos son cuatro, se necesita mostrarles dos objetos, y luego añadir otros dos ante su vista; en el caso de los estudiantes universitarios, estos ya poseen un nivel de abstracción que les permite comprender situaciones con las cuales convive diariamente y establecer conclusiones sobre las mismas, lo cual indudablemente es diferente entre uno y otro estudiante debido a los variados niveles de subjetividad que poseen los estudiantes, los cuales se derivan de los diferentes entornos sociales en los cuales se desenvuelve.

El pensamiento lógico es indispensable para solucionar los problemas cotidianos y para el avance de la ciencia, pues significa sacar conclusiones de las premisas contenidas en ellas, pero no observables en forma directa.

La Lógica es una ciencia universal y formal, que ayuda a realizar razonamientos válidos, pues estudia las formas del pensamiento con independencia de su contenido. Esto es así, ya que el pensamiento lógico busca que, dado un conjunto de premisas, se llegue a una conclusión válida, analizando, comparando, sintetizando luego las partes separadas para el análisis, argumentando las conclusiones a las que se arriba, pues no son productos de la invención, sino que surgen de comprobaciones, lo cual significa que cuando se aplica la lógica se deja sin validez aseveraciones que no cumplan con un proceso válido demostrativo. Para tener un pensamiento lógico se debe partir de enunciados para construir otros correctamente deducidos, independientemente de su veracidad. El objeto de estos razonamientos es la demostración, a la que se llega por deducción. 
Cuando decimos en el lenguaje cotidiano que algo resulta lógico es porque se nos aparece como la conclusión razonable de lo que le antecedió, la idea de las tareas profesionalizantes es desarrollar en los estudiantes universitarios una habilidad que les permita resolver problemas matemáticos de manera lógica y deductiva, aun cuando tenga la necesidad de aplicar complejos algoritmos o utilizar equipos tecnológicos como las computadoras o calculadoras.

Según A. Rincón (1979) se entiende por pensamiento lógico matemático al conjunto de habilidades que cada individuo debe tener para resolver ciertas operaciones básicas, analizar información, hacer uso del pensamiento reflexivo y del conocimiento del mundo que lo rodea, para aplicarlo a su vida cotidiana.

\section{Resolución de problemas}

La resolución de problemas es un proceso mental que supone la conclusión de un proceso más amplio que tiene como pasos previos la identificación del problema y su modelado. Por problema se entiende un asunto del que se espera una solución que dista de ser obvia a partir del planteamiento inicial. Considerada como la más compleja de todas las funciones intelectuales, la resolución de problemas ha sido definida como un proceso cognitivo de alto nivel que requiere de la modulación y control de habilidades más rutinarias o fundamentales en los estudiantes.

La resolución de problemas reside, principalmente, en dos áreas que en muchas ocasiones mantienen una importante correlación debido a las singularidades que las caracterizan: la resolución de problemas matemáticos y la resolución de problemas personales (en los que se presenta algún tipo de obstáculo a su resolución).

La resolución de problemas matemáticos es considerada la parte más esencial de la educación matemática. Mediante la resolución de problemas, los estudiantes experimentan la potencia y utilidad de las matemáticas en el mundo que les rodea, en el caso de los estudiantes universitarios, los problemas matemáticos a solucionar deben mantener coherencia con el entorno laboral de cada profesión, de manera que no estén descontextualizados y afecten la percepción de practicidad lógica que se asume respecto de la profesión en la cual se están formando.

Desde la época de George Pólya (1945) hasta la fecha, son muchos los docentes e investigadores que se han dedicado a buscar respuestas a las dificultades de los estudiantes 
en la resolución de problemas matemáticos, que significa para muchos un placer y para otros una pesadilla, pero lo cierto es que el ser humano no siempre puede evadir el enfrentamiento con ellos, por lo que es necesario desarrollar habilidades para resolverlos.

El desarrollo de las tecnologías de cómputo coloca en primer plano la capacidad de usarla, si se considera su utilización en la resolución de problemas, pero, además, si es utilizada de manera adecuada favorece la asimilación de conocimientos, teniendo en cuenta las posibilidades de acceso a la información que brinda, de procesamiento, de divulgación y socialización, etc.

Sin embargo, los actuales mercados laborales valoran más la utilización eficiente de los equipos tecnológicos sobre los conocimientos teóricos que se posean de la ciencia; las empresas prefieren contratar personas con alta capacidad de resolución de problemas operativos laborales, relegando a un segundo plano a las personas con altos niveles de conocimiento científico.

Por esta razón, la capacidad de resolver problemas se ha convertido en el centro de la enseñanza de la matemática en la época actual, por lo que es necesario contar con una concepción de su enseñanza que ponga en primer lugar la capacidad de resolución de problemas y el desarrollo del pensamiento lógico. A partir de estas ideas centrales es que debe ser determinado el contenido de la enseñanza, por lo que, en ese sentido y dentro de la educación universitaria, resulta pertinente la búsqueda del desarrollo del pensamiento lógico matemático mediante la aplicación de un sistema de tareas profesionalizantes que eviten la utilización de equipos tecnológicos.

En la bibliografía sobre resolución de problemas existen diversas definiciones de problemas, atendiendo cada una a diferentes puntos de vista, aunque diferentes conceptualmente, presentan elementos comunes o al menos no contradictorios. En general, todas coinciden en señalar que un problema es una situación que presenta dificultades para las cuales no hay solución inmediata.

Este problema es muy importante para la didáctica, pues en la selección de los problemas para proponer a un grupo de estudiantes hay que tener en cuenta no solo la naturaleza de la tarea, sino también los conocimientos que las personas requieren para su solución.

Otro aspecto importante a tener en cuenta es que la persona quiera realmente hacer las transformaciones que le permiten resolver el problema, lo que significa que si no está 
motivada, la situación planteada deja de ser un problema al no sentir el deseo de resolverlo; en resumen, en la solución de problemas hay al menos dos condiciones que son necesarias: la vía tiene que ser desconocida y el individuo quiere resolver el problema.

\section{Fases para resolver un problema matemático}

Finalmente, es necesario acotar que para resolver problemas no existen fórmulas mágicas que aseguren contundente que se encontrará la solución; no hay un conjunto de procedimientos o métodos que aplicándolos lleven necesariamente a la resolución del problema, todo depende de la capacidad lógica desarrollada por los estudiantes y por el nivel de interés que mantengan sobre la solución del problema planteado, a pesar de lo cual se puede considerar, como un buen referente, la formulación que hizo Pólya de las cuatro etapas esenciales para la resolución de un problema, que constituyen el punto de arranque de todos los estudios posteriores y actuales relacionados sobre la resolución de problemas.

A continuación, se expone de manera sintética el trabajo de Pólya (1965) que, a criterio del autor del presente trabajo, adolece de la consideración del nivel del nivel de desarrollo del pensamiento lógico que poseen los estudiantes, por lo que las etapas establecidas no surtirían los mismos efectos en todos los estudiantes por las diferencias del sentido lógico citada:

Comprender el problema. Para la comprensión del problema el estudiante tendrá que realizar una lectura detallada, para separar lo dado de lo buscado, lograr hallar alguna palabra clave $u$ otro recurso que permita encontrar una adecuada orientación en el contexto de actuación, expresar el problema con sus palabras, realizar una figura de análisis, establecer analogías entre el problema y otros problemas o entre los conceptos y juicios que aparecen en el texto y otros conceptos y juicios incorporados al saber del individuo, o transferir el problema de un contexto a otro.

Analizar el problema. Para ello, el estudiante deberá analizar nuevamente el problema para encontrar relaciones, precisando e interpretando el significado de los elementos dados y buscados. Los relacionará con otros que puedan sustituirse en el contexto de actuación. Generalizará las propiedades comunes a casos particulares, mediante la comparación de estos sobre la base de la distinción de las cualidades relevantes y significativas de las que no lo son. Tomará decisiones, al tener que comparar diferentes estrategias y procedimientos para escoger el más adecuado. 
Solucionar el problema. Para la realización de esta acción el estudiante deberá: aplicar a la solución de los elementos obtenidos en el análisis del problema.

Evaluar la solución del problema. El sujeto deberá analizar la solución planteada, contemplando diferentes variantes para determinar si es posible encontrar otra solución, verificando si la solución hallada cumple con las exigencias planteadas en el texto del problema.

Es preciso destacar que estas etapas no se dan separadas, aisladas entre sí, sino muy estrechamente unidas con un carácter de espiral, lo cual significa que una fase depende de la anterior para poder ser desarrollada.

Igualmente, es importante señalar que a pesar del abordaje que realiza Pólya en las estrategias a seguir para la resolución de problemas, este no ofrece una clara definición respecto de lo que se considera un problema en el libro "How to solve it"; sin embargo, en el año 1961, con su libro Mathematical Discovery, Pólya evidencia una definición sobre lo que es un problema, y la define aquella situación que requiere la búsqueda consciente de una acción apropiada para el logro de un objetivo claramente concebido, pero no alcanzable de forma inmediata. En otras palabras, una situación, cuantitativa o de otra clase, a la que se enfrenta un individuo o un grupo, que requiere solución, y para la cual no se vislumbra un medio o camino aparente y obvio que conduzca a la misma (Krulik y Rudnik, 1980).

En el año 1966, Pólya brinda un nuevo aporte significativo a la enseñanza de la matemática, en particular, a la resolución de problemas matemáticos y complementariamente al razonamiento lógico numérico a criterio del autor del presente proyecto, con su libro, "Matemáticas y razonamiento plausible", donde expone cómo la construcción matemática puede ser aprovechada para su enseñanza, es decir, cómo las estrategias seguidas por un profesional en matemática, que Pólya denomina "razonamientos plausibles" pueden permitirle a un estudiante aprender matemáticas de manera más rápida y sólida.

De igual manera, Pólya establece un enfoque basado en el desarrollo de estrategias heurísticas, delimita claramente las condiciones que debe poseer un problema para que se derive un aprendizaje significativo, pues sugiere que un problema debe permitirle al estudiante recurrir a problemas análogos para encontrar una solución, realizar conjeturas, generalizar, entre otras. En síntesis, todos los trabajos de Pólya se enfocan en las características fundamentales que debe presentar un problema, así como en el impacto cognitivo que genera la resolución de problemas en los procesos de enseñanza-aprendizaje 
de los estudiantes, entre los cuales se encuentran los estudiantes de las universidades que reciben una formación profesional que los capacita para la adopción de decisiones que tienen repercusiones en los resultados de una organización.

Ante las aseveraciones de Pólya, surge la presencia de Schoenfeld (1985), quien en su libro "Mathematical Problem Solving", considera insuficientes las estrategias planteadas por Pólya para la resolución de problemas y, más bien, sostiene que este proceso es más complejo e involucra más elementos, inclusive de carácter emocional-afectivo, psicológico, sociocultural, entre otros. Schoenfeld establece la existencia de cuatro aspectos importantes que intervienen en todo proceso de resolución de problemas: los recursos (entendidos como conocimientos previos, o bien, el dominio del conocimiento), las heurísticas (estrategias cognitivas), el control (estrategias metacognitivas) y el sistema de creencias.

Los recursos refieren al conocimiento matemático que un estudiante es capaz de brindar en la resolución de un problema, es decir, que para resolver problemas, necesariamente un estudiante debe poseer un nivel de conocimientos que le permitan tal cometido. Las estrategias heurísticas son reglas o planteamientos generales que ayudan en el abordaje de un problema; este aspecto fue ampliamente considerado por Pólya en su libro "Matemáticas y razonamientos plausibles". La manera en que los estudiantes utilizan la información y las estrategias heurísticas que poseen para resolver un problema es lo que Schoenfeld denomina control, este involucra conductas de interés tales como: planificar, seleccionar metas y submetas y monitoreo constante durante el proceso de resolución.

Finalmente, Schoenfeld establece un aspecto transversal en la resolución de problemas y lo denomina sistema de creencias, que consiste en el conjunto de ideas o percepciones que los estudiantes poseen a cerca de la matemática y su enseñanza, es decir la subjetividad que posee una persona en relación con los problemas matemáticos, está comprobado que si un estudiante piensa que resolver un problema es una actividad difícil y compleja, pues seguramente tendrá complicaciones en entender un problema, plantear un algoritmo para solucionarlo y encontrar la solución real y lógica.

Es necesario acotar que en todas las ideas de Pólya y Schoenfeld relacionadas con la resolución de problemas matemáticos mediante la aplicación de habilidades lógicas desarrolladas en un estudiante, no contemplan la intervención de equipos y elementos tecnológicos, y se debe a que los autores realizaron sus respectivos trabajos investigativos en 
épocas y situaciones en las cuales no existía una alta influencia de la tecnología en los procesos de enseñanza aprendizaje.

Actualmente, la situación que se vive en las aulas universitarias es diferente y se evidencia con la masiva presencia de equipos tecnológicos que utilizan los estudiantes como herramientas dentro de su formación profesional, por lo que resulta viable el replanteo de las ideas de los autores citados, para enmarcarlas dentro de un entorno pedagógico poblado de tecnología y un entorno laboral lleno de demandas de habilidades relacionadas con el dominio de equipos y programas tecnológicos para un desenvolvimiento profesional óptimo.

El razonamiento lógico matemático, que se desarrolla en los estudiantes universitarios, mantiene un grado de influencia evidente de la tecnología, por lo que resulta estratégicamente pedagógico plantearse en los diferentes niveles de estudios superiores un conjunto de tareas profesionalizantes que observen el uso gradual y secuencial de los equipos tecnológicos necesarios para desarrollar capacidades que les permitan insertarse a los egresados de las universidades en el campo laboral, sin que este uso signifique un obstáculo para el aumento de la capacidad lógica resolutiva que deben poseer las personas dentro de un entorno socio económico

\section{Síntesis y reflexiones finales}

Existen varias razones que pueden ser utilizadas por el profesor universitario en su estrategia para la motivación de sus estudiantes en relación con el desarrollo del pensamiento lógico matemático, como: el papel de la solución de problemas matemáticos en situaciones de la vida, el papel que ha desempeñado la matemática, en general, y la solución de problemas en particular, en el propio desarrollo de la historia de la matemática como ciencia y la función desarrolladora de los problemas y su contribución al desarrollo intelectual del futuro profesional y específicamente sobre la formación de su pensamiento.

Las motivaciones en este campo son llamadas motivaciones extra matemáticas, para que resulten verdaderamente interesantes los problemas deben representar situaciones actuales y del entorno, ajustarse estrictamente a la realidad, no requerir de equipos tecnológico para su solución y ser asequibles para los estudiantes, sin perder de vista que las dificultades que se incluyan deben ir aumentando periódicamente para efectos del entrenamiento progresivo del pensamiento lógico matemático que se pretende desarrollar en 
los estudiantes como una función de fundamento de la posterior actuación laboral. A manera de conclusiones, el autor del trabajo asume las siguientes:

- El desarrollo del pensamiento lógico, la solución de problemas matemáticos y la invención de equipos tecnológicos están interrelacionados de manera directamente proporcional y positiva.

- Los estudiantes que tienen acceso fácil a equipos tecnológicos educativos demuestran mayor dependencia para solucionar problemas matemáticos, lo cual es un obstáculo para el desarrollo de sus capacidades de razonamiento lógico.

- Los estudiantes con dificultades para adquirir equipos tecnológicos demostraron mayor capacidad de razonamiento lógico en las pruebas de solución de problemas y esto se debe a la obligada actitud de dependencia personal que poseen.

- La motivación es importante para el desarrollo de la capacidad de solución de problemas que tienen los estudiantes, lo cual se evidenció con mayor incidencia en los estudiantes de las universidades estatales, quienes demostraron mayor interés e importancia a los contenidos y conocimientos adquiridos como parte de su formación profesional.

- Es necesario que los estudiantes ejerciten el pensamiento lógico matemático mediante un sistema de tareas profesionalizantes que les permita solucionar problemas con o sin la utilización de equipos tecnológicos.

\section{Referencias}

Asimov, Issac. (2003). Momentos estelares de la ciencia. Madrid, España: Alianza Editorial.

Guilford, Joy Paul. (1977). La naturaleza de la inteligencia humana. Buenos Aires, Argentina: Editorial Paidos.

Krulik, Stephen y Rudnick, Jesse. (1980). Problem solving in school mathematics. National council of teachers of mathematics. Year Book. Virginia: Reston

Palacios Frugone, Miguel. (2010). La psicología de un hombre llamado Jesús. Guayaquil, Ecuador: Ediciones Cultura y Didáctica S.A.

Pólya, George. (1945). How to solve it. Princeton, NJ, Estados Unidos: Princeton University Press.

Pólya, George. (1961). Mathematical Discovery: On Understanding, Learning and Teaching Problem Solving (Combined Edition). Cleveland, Ohio: John Wiley \& Sons. 
Pólya, George. (1965). Cómo Plantear y Resolver Problemas. México: Editorial Trillas

Pólya, George. (1966). Matemáticas y razonamiento plausible. Madrid: Tecnos.

Rincón, Ana. (1979). Desarrollo del pensamiento lógico matemático. Bogotá, Colombia: Editorial Planeta.

Schoenfeld, Alan H. (1985). Mathematical problem solving. Orlando, FL: Academic Press. 